\title{
Measurement of stratospheric $\mathrm{HBr}$ using high resolution far infrared spectroscopy
}

\author{
M. Carlotti, ${ }^{1}$ P. A. R. Ade, ${ }^{2}$ B. Carli, ${ }^{3}$ P. Ciarpallini, ${ }^{4}$ U. Cortesi, ${ }^{3}$ M. J. Griffin, ${ }^{2}$ \\ G. Lepri, ${ }^{1}$ F. Mencaraglia, ${ }^{3}$ A. G. Murray, ${ }^{2}$ I. G. Nolt, ${ }^{5}$ J. H. Park, ${ }^{5}$ \\ and J. V. Radostitz ${ }^{6} \dagger$
}

\begin{abstract}
Far infrared spectral features of $\mathrm{HBr}$ have been observed in the stratospheric emission spectrum using a balloon borne high resolution Fourier transform spectrometer equipped with a high sensitivity detector specially designed for this purpose. The value of $1.6 \pm 0.6$ parts per trillion in volume for the $\mathrm{HBr}$ mixing ratio has been retrieved, from the global-fit analysis of 121 spectra, in the $25-36.5 \mathrm{~km}$ altitude range. The result is briefly compared with models and previous assessments.
\end{abstract}

\section{Introduction}

The importance of bromine chemistry in the processes that lead to the depletion of the ozone layer has been identified [World Meteorological Organization, 1992]. The catalytic cycles involving bromine are thought to cause an ozone depletion equivalent to that of the better known chlorine cycles, although the bromine concentration is only a few percent of that of chlorine [MC Elroy et al., 1992]. In this context, a specific concern has been raised on the impact of the anthropogenic release in the atmosphere of methyl bromide $\left(\mathrm{CH}_{3} \mathrm{Br}\right)$ which is extensively used as an agricultural soil fumigant and as a general pesticide.

In the middle atmosphere, $\mathrm{HBr}$ is a reservoir species of

\footnotetext{
${ }^{\dagger}$ Deceased Oct. 6, 1993. We dedicate this report of our work to the memory of our colleague whose techmical skills contributed greatly to this program. Italy.

${ }^{1}$ Dipartimento di Chimica Fisica ed Inorganica, Università di Bologna,

${ }^{2}$ Queen Mary and Westfield College, London, UK.

${ }^{3}$ Istituto di Ricerca sulle Onde Elettromagnetiche del C. N. R. "Nello Carrara", Firenze, Italy.

${ }^{4}$ Dipartimento di Astronomia e Scienza dello Spazio, Università di $\mathbf{F l}_{\mathbf{1}}$ renze, Italy

${ }^{5}$ National Aeronautics and Space Administration, Langley Research Center, Hampton, Virginia.

${ }^{6}$ University of Oregon, Eugene, Oregon.
}

Copyright 1995 by the American Geophysical Union.

Paper number 95GL03264

0094-8534/95/95GL-03264\$03.00 the bromine chemistry and its measurement is needed to check the validity of current theoretical models. However, the low abundance of this molecule is such that the attempts to measure its concentration have not provided up to now an unambiguous result. Park et al. [1989] have reported the only positive detection of $\mathrm{HBr}$ in the stratosphere with a mixing ratio of $20 \pm 7$ parts per trillion in volume (pptv) based on 1979 measurements, but this result has not been confirmed by two subsequent measurement campaigns carried out in 1982 and 1992 with the same instrument. Traub et al. [1992] have determined an upper limit of $4 \mathrm{pptv}$ at $32 \mathrm{~km}$ of altitude, combining the observations of three different measurement campaigns. Their data have been more recently analysed together with data of four additional campaigns to provide an average mixing ratio of $2.0 \pm 0.8 \mathrm{pptv}$ in the altitude range of 22 to $34 \mathrm{~km}$ [Johnson et al., 1995]. The experimental disagreement made it evident that a better measurement sensitivity was needed.

In this paper we report the results of measurements obtained in the far-infrared spectral region with a balloon-borne high resolution Fourier transform spectrometer having a specially designed spectral channel for the detection of $\mathrm{HBr}$.

\section{Measurements}

The instrument was optimized for the measurement of the $\mathrm{J}=3 \rightarrow 2$ pure rotational transition of the two $\mathrm{HBr}$ molecular species that correspond to the 79 and 81 bromine isotopes. Due to the coupling between the nuclear spin and the molecular rotation, each of these transitions has a well known hyperfine structure that corresponds to the values of the total-angular-momentum quantum number $\mathrm{F}$ [Di Lonardo et al., 1991]; the envelope of these structures results in two spectral features peaked at 50.054 and $50.069 \mathrm{~cm}^{-1}$. Since the two bromine isotopes have approximately equal natural abundance, the two spectral features are of equal intensity. The selection of the $J=3 \rightarrow 2$ transitions was based on both their occurrence within a relatively transparent window of the atmospheric spectrum and the coincidence with the maximum of the Boltzmann distribution, which, for $\mathrm{HBr}$ at the average stratospheric temperature of $250 \mathrm{~K}$, gives $\mathrm{J}=3$ as the most populated level. The spectrometer used for the measurements is a Martin-Puplett type interferometer capable of $0.0025 \mathrm{~cm}^{-1}$ unapodized spectral resolution. It is an improved version of the instrument, described in Carli et al. [1984], that was used in the work [Park et al., 1989] reporting the previous detection of $\mathrm{HBr}$. The interferometer measures the difference between the signals entering two input ports; one of the inputs observes the Earth's limb while the other 
is oriented toward the zenith. An advanced photoconductive detector was designed for these measurements with cold optical filters that limit the bandwidth to an interval of about $2 \mathrm{~cm}^{-1}$ [Murray et al., 1992]. Such a narrow frequency interval minimizes the photon noise in the incident power on the detector resulting in a single scan Signal-to-Noise Ratio (SNR) of better than 500 relative to a unit emissivity defined as the flux observed from a $250 \mathrm{~K}$ blackbody. Each full resolution scan of $100 \mathrm{~cm}$ mirror stroke required about $2.7 \mathrm{~min}$. The instrument was launched from Ft. Sumner, NM (latitude $34^{\circ} \mathrm{N}$, longitude $104^{\circ} \mathrm{W}$ ) on May 31,1993 reaching the float altitude of $36.5 \mathrm{~km}$ at about $0800 \mathrm{hrs}$ (local solar time). The thermal emission of the atmosphere was continuously measured with limb-scanning observations for a duration of about 20 hours. However, due to a loss in altitude in the latter part of the flight and to the altitude range of the reported results (see below), we have not used in the analysis data obtained after midnight (local solar time) when the balloon dropped below $25 \mathrm{~km}$.

\section{Analysis}

The available data were screened to select the spectra in which signals at the $\mathrm{HBr}$ frequencies were not affected by an aliased frequency signal coming from a spurious electronic pickup; a total of 121 spectra resulted from the selection. Figure 1 shows the $\mathrm{HBr}$ frequency window for six individual spectra corresponding to observations at the tangent altitudes of $21.0,22.8,24.8,26.6,28.3$, and $33.4 \mathrm{~km}$ respectively. The spectra are linearly apodized, to reduce the effect of the instrument function side-lobes, and are normalized with respect to the emission of a blackbody at $286 \mathrm{~K}$; the two vertical bars mark the frequency position where $\mathrm{HBr}$ transitions occur (similar bars are used in the figures that follow). Despite the high SNR achieved, Fig. 1 shows that no spectral feature can be positively assigned to $\mathrm{HBr}$ transitions in spectra corresponding to a single observation. Enhancement of the SNR can be obtained through spectral averaging.

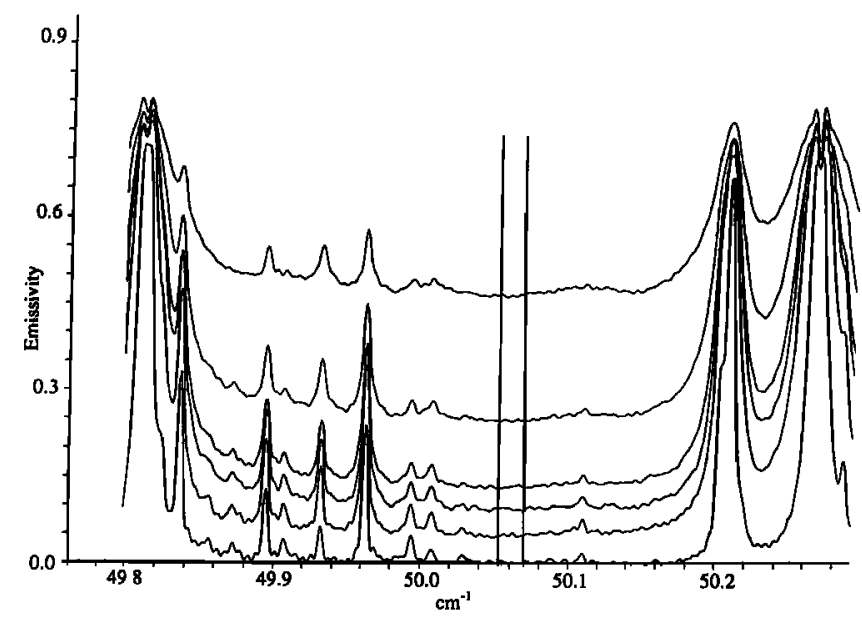

Figure 1. Sample of 6 limb-scanning spectra. The tangent altitude of the line of sight corresponding to these spectra is $21.0,22.8,24.8,26.6,28.3$, and $33.4 \mathrm{~km}$ starting from top. The vertical bars mark the frequency position where $\mathrm{HBr}$ transitions occur (similar bars are used in all figures).
However it results that, for an useful SNR enhancement, also measurements with different observation geometry must be averaged, making difficult the quantitative analysis of such averages because of the non-linear dependence of the spectra on the observation-geometry parameters. We therefore decided to perform a global-fit analysis [Carlotti, 1988] of the individual spectra: i.e. all 121 spectra were fitted simultaneously but each with a synthetic spectrum calculated with the corresponding observation geometry. A non-linear least squares (NLS) procedure was used to determine only the altitude distribution of $\mathrm{HBr}$ mixing-ratio. The altitude distribution profile of ozone, which is needed to model the nearby atmospheric features, was previously determined and used as a known input in the $\mathrm{HBr}$ analysis. The atmospheric line database used for modelling the simulated spectral features was the 1992 release of HITRAN [Rothman et al., 1992], complemented with additional spectroscopic data from the release 3 of the JPL line compilation catalogue [Poynter and Pickett, 1985] and the SAO line database [Chance et al., 1994]. The temperature and pressure altitude distributions were measured by radiosondes launched, at the time of the experiment, within the same airmass as observed by the spectrometer.

The quality of this global fit can be represented by means of average spectra. Figure 2 shows the apodized average of the 121 spectra in the frequency interval where the simulations have been performed. The box-delimited portion of Fig. 2 is expanded for clarity in Fig. 3. In this figure a further box indicates the frequency interval over which the fit was made. The simulation is performed for intervals outside the fitted interval in order to correctly account for the side-lobes of the instrument function. Figure 4 shows the quality of the fit and highlights the identification of $\mathrm{HBr}$ features; in this figure curve 1 is a blow up of the marked portion of Fig.

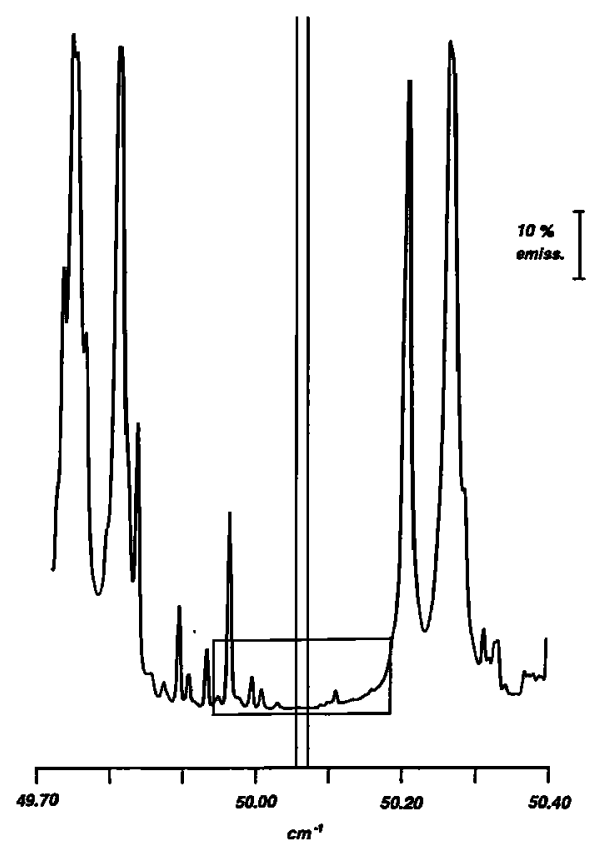

Figure 2. Average of the 121 analyzed spectra in the frequency interval where the simulations have been performed. The box delimits, in the frequency scale, the interval shown in the blow up of Fig. 3. 


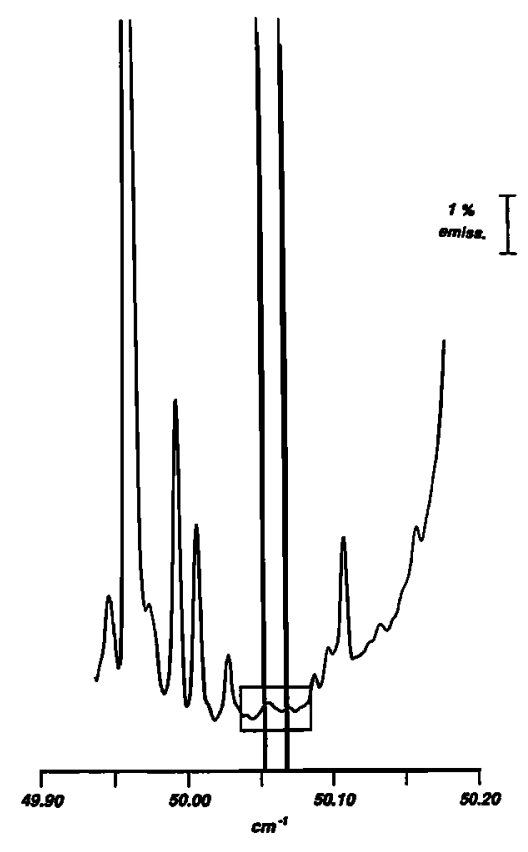

Figure 3. An enlargement of the marked portion of Fig. 2. The box delimits, in the frequency scale, the interval where the residuals were minimized by the NLS procedure (see text).

3 , curve 2 is the average of the 121 simulated spectra after convergence has been reached by the fitting procedure, curve 4 is the difference between curve 1 and curve 2, curve 3 is an average of simulated spectra, as in curve 2 but in the case of no $\mathrm{HBr}$, curve 5 is the difference between curve 2 and curve 3 . In Fig. 4 the vertical bars show a splitting due to the position of the two main components of the $\mathrm{HBr}$ hyperfine structure, that become discernible at this expansion of the frequency scale. Curve 3 of Fig. 4 highlights the presence of an interfering transition, due to isotopically substituted ozone, which overlaps with the lower frequency $\mathrm{HBr}$ feature. The residual of the fit shown by curve 4 of Fig. 4 is larger than what could be expected from purely random spectral noise. Curve 4 is actually dominated by systematic effects due to our limit in the modelling of both the instrument line shape (periodic oscillation) and the wings of nearby atmospheric lines (slope). We have not used a parametric model for these effects since the possible improvement in the fit would have been obtained at the expense of a larger systematic error.

We decided to adopt $25 \mathrm{~km}$ as the lowest value for the tangent altitude for the observations included in this analysis because the addition to the fitted data-set of lower tangent altitudes resulted in slightly higher values for the r.m.s. deviation of curve 4 of Fig. 4, with no apparent change in the retrieved value of the mixing ratio.

\section{Results}

Attempts to determine the distribution of $\mathrm{HBr}$ as a function of altitude led to large uncertainties on the retrieved mixing ratio values. Even if exploiting the trade-off between the uncertainty of the retrieved profile and its vertical reso-

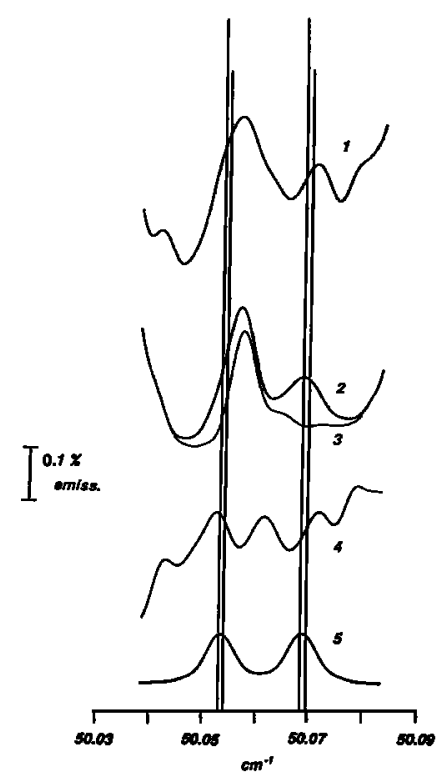

Figure 4. Curve 1 is an enlargement of the boxed experimental average of Fig. 3. Curve 2 is the average of the corresponding 121 simulated spectra after convergence has been reached by the global fitting algorithm, and curve 4 is the residual difference between curve 1 and curve 2 . Curve 3 is the average of simulated spectra as in curve 2 but in with no $\mathrm{HBr}$ included in the calculations, so that curve 5 which is the difference between curve 2 and curve 3 , represents the spectral component attributed to $\mathrm{HBr}$ emission.

lution [Carlotti et al., 1994] in favour of the first quantity, the uncertainty on the retrieved mixing ratios could not be reduced to useful values. Therefore, on the basis of existing photochemical models (I. Isaksen, personal communication, 1994) a constant $\mathrm{HBr}$ mixing ratio profile was assumed in the fitting procedure and results in the value of $1.63 \mathrm{pptv}$ with a $1 \sigma$ error of 0.51 pptv that corresponds to $31 \%$ of uncertainty. This error is the Estimated Standard Deviation (ESD) derived from the variance-covariance matrix of the fit [Carlotti et al., 1994] and the r.m.s. deviation of the residuals which are shown in curve 4 of Fig. 4 . The uncertainty of $31 \%$ is therefore the random error component due to spectral noise and to the modelling limits mentioned in the previous section.

The systematic error sources, accompanied in parentheses by the corresponding percent value of the error are: spectroscopic parameters of the $\mathrm{HBr}$ transitions (3\%), interfering ozone transition $(10 \%)$, finite discretizations in the forward model computation (3\%), starting value of the $\mathrm{HBr}$ mixing ratio for the retrieval process $(5 \%)$, observation geometry $(8 \%)$, temperature and pressure altitude distributions $(4 \%)$, intensity calibration ( $4 \%)$. The squared summation of all the error components provides the value of $35 \%$ for the overall error, that corresponds to $0.56 \mathrm{pptv}$.

The current result of $1.6 \pm 0.6 \mathrm{pptv}$ for $\mathrm{HBr}$ mixing ratio in the $25-36.5 \mathrm{~km}$ altitude range is consistent with the average value of $2.0 \pm 0.8$ pptv obtained by Johnson et al. [1995] but is in large disagreement with the measurement of $20 \pm 7 \mathrm{pptv}$ obtained by means of the same transition and with an earlier 
version of the current instrument in 1979 [Park et al., 1989]. Since a large variability of $\mathrm{HBr}$ concentration is improbable, the explanation for the result of 1979 measurements must be found in either a local contamination within the gondola environment or in an instrumental error. No realistic source of local contamination has been identified. A comparison of the 1979 spectra with those obtained in 1993 with better SNR shows no significant differences apart from the intensity of the $\mathrm{HBr}$ features. The local character of the differences and the long time passed make it impossible to identify the possible cause of instrumental errors.

The mixing ratio measured in this work is greater than anticipated by the theoretical model by Isaksen (I. Isaksen, personal communication, 1994) and is consistent with the model by Lary (D. J. Lary, paper submitted to J. Geophys. Res., 1995) in the case in which a fraction greater than $1 \%$ of the reaction of $\mathrm{HO}_{2}$ with $\mathrm{BrO}$ yields $\mathrm{HBr}$. This fraction of $\mathrm{HBr}$ production is considered to be high even if it is not precluded by laboratory measurements [e.g., Poulet et al., 1992]. The measured mixing ratio indicates that a significant fraction of the total stratospheric bromine is not in reactive form and implies that improvements are needed in the understanding of bromine photochemistry.

Acknowledgments. We gratefully acknowledge the support of this work by the Methyl Bromide Global Coalition. The balloon flight program at NASA Langley is supported by the Correlative Measurements Program of the Upper Atmosphere Research Satellite and the Upper Atmosphere Research Program Office. In Italy, the study received support by the Consiglio Nazionale delle Ricerche, and the Italian Space Agency (ASI). In UK support has been provided by the Natural Environment Research Council grant GR/J54987. Flight launch assistance was provided by the Balloon Support Group of the Jet Propulsion Laboratory and the National Scientific Balloon Facility, both which operate under contract to NASA.

\section{References}

Carli, B., F. Mencaraglia, and A. Bonetti, Sub-millimeter high resolution FT spectrometer for atmospheric studies, Appl. Opt., 23, 2594-2603, 1984.

Carlotti, M., Global-fit approaa $b$ to the analysis of limb-scanning atmospheric measurements, Appl. Opt., 27, 3250-3254, 1988.

Carlotti, M., and B. Carli, Approach to the design and data analysis of a limb-scanning experiment, Appl. Opt., 33, 3237-3249, 1994.

Chance, K. V., K. W. Jucks, D. G. Johnson, and W. A. Traub, The Smithsonian Astrophysical Observatory database SAO92, J. Quant. Spectrosc. Radiat. Transfer, 52, 445-457, 1994.
Di Lonardo, G., L. Fusina, P. De Natale, M. Inguscio, and M. Prevedelli, The pure rotation spectrum of $\mathrm{HBr}$ in the Sub-mm region, J. Mol. Spectrosc., 148, 86-92, 1991.

Johnson, D. G., W. A. Traub, K. V. Chance, and K. W. Jucks, Remote sensing measurements of $\mathrm{HBr}$ and $\mathrm{HOBr}$ and implications for bromine partitioning in the stratosphere, Geophys. Res. Lett., 22, 1373-1376, 1995.

Mc Elroy, M. B., R. J. Salawitch, and K. Minschwaner, The changing stratosphere, Planet. Space Sci., 40, 373-401, 1992.

Murray, A. G., M. J. Griffin, P. A. R. Ade, J. Leotin, C. Meny, and G. Sirmain, Optimisation of FIR photodetectors for Atmospheric Spectroscopy, ESA SP-356, 159-163, 1992.

Park, J. H., B. Carli, and A. Barbis, Stratospheric $\mathrm{HBr}$ mixing ratio from far infrared emission spectra, Geophys. Res. Lett., 16, 787-790, 1989.

Poulet, G., M. Pirre, F. Maguin, R. Ramarosou, and G. Lebras, The role of $\mathrm{BrO}+\mathrm{HO}_{2}$ reaction in the stratospheric chemistry of Bromine, Geophys. Res. Lett., 19, 2305-2308, 1992.

Poynter, R. L., and H. M. Pickett, Submillimeter, millimeter, and microwave spectral line catalogue, Appl. Opt., 24, 2235-2240, 1985.

Rothman, L. S., R. R. Gamache, R. H. Tipping, C. P. Rinsland, M. A. H. Smith, D. Chris Benner, V. Malathy Devi, J. M. Flaud, C. Camy-Peyret, A. Perrin, A. Goldman, S. T. Massie, L. R. Brown, and R. A. Toth, The HITRAN molecular database: editions of 1991 and 1992, J. Quant. Spectrosc. Radiat. Transfer, 48, 469-502, 1992.

Traub, W. A., D. G. Johnson, K. W. Jucks, and K. V. Chance, Upper limit for stratospheric $\mathrm{HBr}$ using far-infrared thermal emission spectroscopy, Geophys. Res. Lett., 19, 1651-1654, 1992.

World Meteorological Organization, Global Ozone Research and Monitoring Project, Scientific assessment of ozone depletion, Report No. 25, WMO, Geneva, Switzerland, 1992.

M. Carlotti and G. Lepri, Dipartimento di Chimica Fisica ed Inorganica, Università di Bologna, Viale del Risorgimento 4, 40136, Bologna, Italy

P. A. R. Ade, M. J. Griffin, and A. G. Murray, Queen Mary and Westfield College, Mile End Rd., E1 4NS London, UK

B. Carli, U. Cortesi and F. Mencaraglia, Istituto di Ricerca sulle Onde Elettromagnetiche "Nello Carrara" del C.N.R., Via

Panciatichi 64, 50127 Firenze, Italy

P. Ciarpallini, Dipartimento di Astronomia e Scienza dello

Spazio, Università di Firenze, Gruppo Fisica Cosmica e

Aeronomia, Via Leone Pancaldo 3/37, 50127 Firenze, Italy

I. G. Nolt and J. H. Park, NASA Langley, Hampton, VA, 23681-0001 USA

(received April 24, 1995;

revised October 16, 1995;

accepted October 16,1995.) 